\title{
Subproblem analysis of discrimination shift learning in the pigeon*
}

\author{
THOMAS J. TIGHE and VIRGIL GRAF \\ Dartmouth College, Hanover, N.H. 03755
}

Pigeons were given reversal and extradimensional shifts within a two-dimension two-pair discrimination task. Analysis of learning on the individual stimulus pairs during shifts indicated that the Ss learned the pairs as independent subproblems rather than as instances of a single problem.

This paper reports an extension of the subproblem analysis of discrimination shift learning (Tighe, Glick, \& Cole, 1971) to a previously untested species. The point of the subproblem analysis is to determine whether the stimulus instances of a shift task have been learned as independent subproblems rather than as instances of a single problem. Application of the analysis requires an extradimensional (ED) shift involving the same stimuli as in preshift training. Under this procedure, the stimulus-reward relations of preshift training are maintained for half of the task instances, but reversed for the other half. If Ss do learn the task instances independently, only those instances which undergo change in reward relations should pose relearning problems. Independence in subproblem learning, then, is measured by the difference between the learning functions on the changed and unchanged instances.

Tighe et al (1971) applied the subproblem analysis to data from a comparison of reversal $(R)$ and ED shifts in children trained in a two-dimension, two-pair concept task. They found that 10-year-olds exhibited similar learning functions on unchanged and changed pairs of ED shift and high error rates on both pairs. These Ss accomplished $R$ shift faster than ED shift. In contrast, Graf \& Tighe (1971) observed complete independence in subproblem learning by turtles trained in similarly structured tasks. In an ED shift, these Ss maintained errorless performance on the unchanged stimulus pair throughout relearning of the reversed pair. Similarly, Tighe \& Frey (1972) found marked independence in subproblem learning by rats trained in an R-ED shift comparison. For the turtles and rats, ED shift was easier than $R$ shift.

*This research was supported by Grants EY-00355 and MH-11088 from the National Institutes of Health. Thomas Tighe is supported by Research Career Development Award K4-HD-43, 859 from the National Institute of Child Health and Human Development. We gratefully acknowledge the assistance of Bruce A. Douglas.
The present experiment sought further evidence on the generality of independent subproblem learning in infrahuman $\mathrm{Ss}$ and on the relation between pattern of subproblem learning and relative ease of $R$ vs ED shifts.

\section{SUBJECTS}

The Ss were 16 6-month-old male White Carneaux pigeons obtained from the Palmetto Pigeon Plant (Sumter, South Carolina). The Ss were maintained at $80 \%$ of their free-feeding weights throughout training. Due to space limitations, Ss were trained in two squads (composed of 7 and $9 \mathrm{Ss}$ ) over successive 3-month periods.

\section{APPARATUS}

The apparatus was a Lehigh Valley pigeon chamber (Model 132-02) equipped with a Lehigh Valley 141-10 intelligence panel. Contingencies were automatically controlled with electromagnetic relays, timers, and steppers.

\section{The PROCEDURE}

received conventional magazine and keypeck training and discrimination which required Ss to choose between two differentially illuminated response keys. The keys were illuminated with red or green light which was either steady or flickering. Flicker stimuli were obtained by routing the power which energized the stimulus lamps through a 15- $\mathrm{Hz}$ multivibrator (Grason-Stadler E783F pulse former). The stimulus pairs which comprised the discrimination task (red-steady vs green-flicker, and red-flicker vs green-steady) were presented according to a Fellows sequence with regard to order of pair presentation and left-right position of the pair members (Fellows, 1967). Each S received 20 trials per day involving 10 presentations of each stimulus pair. If $S$ made a correct response to a stimulus pair, he received a 4-sec grain reinforcement, followed by a 6-sec time-out period, after which the next trial (stimulus pair) was presented. If $S$ made an error, he received a $12-\mathrm{sec}$ time-out period, followed by presentation of the next stimulus pair.
The Ss were trained in the initial discrimination to a criterion of 18 correct responses on each of 2 successive days. On the day following attainment of criterion, half the animals received an $\mathrm{R}$ shift and half an ED shift. For example, an $\mathrm{S}$ who had red as $\mathrm{S}+$ in the initial discrimination could be given an $R$ shift to green as $\mathrm{S}+$, or an ED shift to steady or flicker as $\mathrm{S}+$. Shift training was identical in all procedural respects to that of the initial discrimination and was continued to the aforementioned criterion. Red, green, steady, and flicker were used equally often as the $\mathrm{S}+$ in both shift conditions, and Ss were assigned randomly to shift and $\mathrm{S}+$ conditions.

\section{RESULTS}

Analysis of error and trial scores yielded virtually identical conclusions, and hence only the error analyses will be presented here.

Initial Discrimination

The mean number of errors to criterion for the subgroups trained with hue relevant and later placed in $R$ and in ED shift were 17.2 and 15.2, respectively; the mean errors for subgroups trained with steady-flicker cues relevant and later placed in $R$ and ED shift were 46.5 and 24.7, respectively. Factorial analysis of these data showed that learning was easier when hue was relevant than when steady-flicker cues were relevant $(\mathrm{F}=$ $35.7, \mathrm{df}=1 / 12, \mathrm{p}<.001)$, that $\mathrm{Ss}$ assigned to the ED condition made fewer errors than those assigned to the $\mathrm{R}$ condition $(\mathrm{F}=13.4, \mathrm{df}=1 / 12$, $p<.005$ ), and that the interaction between dimension and shift assignment was significant $(F=9.26$, $\mathrm{df}=1 / 12, \mathrm{p}<.025)$. Analysis of the interaction by $t$ tests showed that $S s$ assigned to ED shift had fewer errors than those assigned to $\mathrm{R}$ shift under the steady-flicker relevant condition ( $t$ $=4.93, \mathrm{df}=6, \mathrm{p}<.005$ ), but $\mathrm{Ss}$ assigned to $R$ and ED conditions did not differ in errors to criterion under the hue relevant condition $(t=.42, \mathrm{df}$ $=6, \mathrm{p}>.50$ ).

\section{Shift Learning}

The mean number of errors to criterion in $R$ and ED shifts with hue relevant were 77.0 and 35.5 , respectively; the mean errors in $R$ and ED shifts with steady-flicker relevant were 134.5 and 49.5 , respectively. Factorial analysis of this measure revealed that $E D$ shift was learned more readily than $\mathrm{R}$ shift $(\mathrm{F}=\mathbf{2 0 . 6}$, df $=1 / 12, p<.001)$, and that there were fewer errors when hue was relevant than when steady-flicker cues were relevant $(F=6.59, \mathrm{df}=1 / 12$, $p<.025)$. The $F$ value for the interaction of dimension and shift task did not approach significance.

Since there were lower error scores 


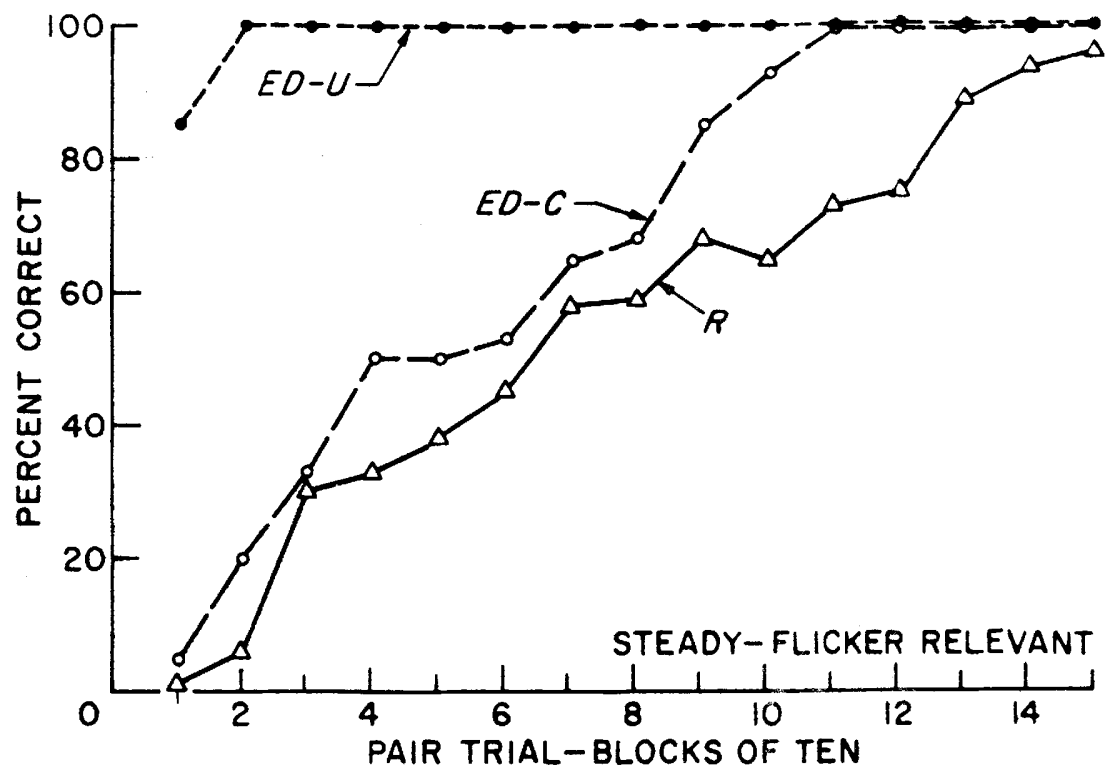

Fig. 1. Learning functions on ED-C, ED-U, and R shift with steady-flicker relevant. For $\mathrm{R}$ shift, pair trial refers to successive presentations of both stimulus pairs.

among $\mathrm{ED}$ shift $\mathrm{Ss}$ as compared to $\mathrm{R}$ shift Ss in the initial discrimination as well as in the shift phase, the difference between $R$ and ED learning could be due to the chance assignment of superior learners to the ED condition. To assess this possibility, the $R$ vs $E D$ difference was evaluated by analysis of covariance (Edwards, 1968) applied to the error scores of the initial and shift discriminations collapsed over dimensions. This analysis showed that with adjustment for the difference in initial learning the ED group still made significantly fewer errors than the $R$ group $(F=$ 8.38 , df $=1 / 13, p<.02)$. Thus, the superiority of $E D$ over $R$ shift learning does not depend upon difference in the nature of the Ss assigned to $R$ and ED groups.

$$
\text { Subproblem Analysis }
$$

The data of major concern in the experiment are shown in Figs. 1 and 2, which depict shift learning in terms of the subproblem analysis. In interpreting these figures, it should be recalled that an ED shift involves reversal of the stimulus-reward relations on one of the stimulus pairs, the other pair retaining the reward relations which obtained in the initial discrimination. Thus, for example, an $\mathbf{S}$ who is shifted from red as $\mathrm{S}+$ to steady as $\mathrm{S}+$ would continue to be rewarded for choosing red-steady as opposed to green-flicker, but would have to learn to reverse response to the pairing of red-flicker vs green-steady. In $R$ shift, stimulus-reward relations are reversed for both of the pairs. The graphs show the group learning functions for the changed pair in ED their shift problem while concurrently exhibiting a high reversal-like error rate on the other pair of the same problem. Three of the four birds given the ED problem with steady-flicker relevant never made an error on the unchanged pair at any time during shift learning. The remaining bird made errors on ED-U only on the first day of shift and then exhibited errorless performance on this pair through the 4 remaining days of his training. Similarly, two of the four birds given the ED problem with hue relevant never made an error on ED-U during shift, while the daily performances of the remaining two birds never fell below $80 \%$ correct choice on ED-U.

It is notable that within each condition of dimension relevant the error rate on the changed pair of the ED problem closciy approximated the error rate for the pairs of $R$ shift. Essentially, $R$ shift posed two reversal subproblems, while ED shift posed one reversal subrob'em, each of which was learned at about the same rate.

\section{DISCUSSION}

The pattern of subproblem learning observed in th present study is strikingly similar to that found in the previously cited experiments with rats and turtles. Taken together, these studies strongly suggest that independent subproblem learning may be the usual solution mode by infrahuman $\mathrm{Ss}$ within the R-ED paradigm. Consequently, $R$ shift should be relatively difficult for such Ss, and current review (Kendler, 1971) indicates that this is the case.

Independence in subproblem learning, as observed herein, is unexpected from the viewpoint of

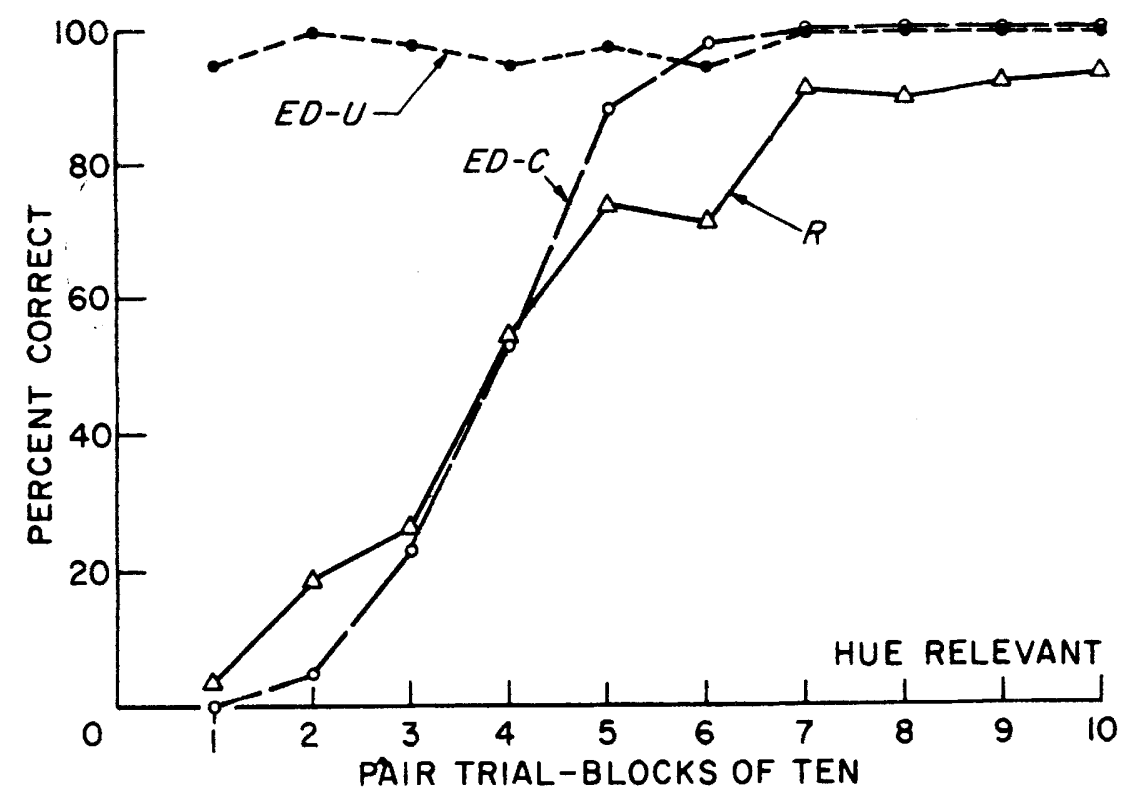

Fig. 2. Learning functions on ED-C, ED-U, and $R$ shift with hue relevant. For $R$ shift, pair trial refers to successive presentations of both stimulus pairs. 
attention theory (e.g., Sutherland, 1959; Zeaman \& House, 1963), which assumes that discrimination learning in all Ss proceeds by selective sampling of the task dimensions and that attention to the relevant dimension is prerequisite to development of correct choice behavior. Under this view, solution of the ED problem requires extinction of attention to the initially relevant dimension and the strengthening of attention to the formerly irrelevant dimension. Such redirection of attention would necessarily affect performance on ED-U. More specifically, nonreinforcement on ED-C should weaken the attentional response which is linked to choice on both ED-C and ED-U and consequently should disrupt performance on ED-U. Such disruption may or may not occur early in shift, but would certainly be expected as correct choice on ED-C reaches the chance level. Moreover, at this point a period of chance responding on ED-U and ED-C would be expected since following extinction of attentional response to the formerly $r$ elevant dimension S s would have an initial likelihood of sampling both relevant and irrelevant cues. But there is no suggestion of such behavior changes in Figs. 1 and 2; rather, the pigeons appear to have simply shifted choice response on the specific stimulus compounds affected by the altered reinforcement contingencies.
The subproblem behavior of the pigeons is surprising in view of data attesting to dimension selectivity in visual discrimination learning by pigeons. For example, Reynolds (1961) reported that pigeons learned about only one of two dimensions within a redundant relevant-cue paradigm. Ss were trained to discriminate between two compounds-a white triangle on a red background (the $S+$ ) and a white circle on a green background (the $\mathbf{S}-$ ). When the birds were later tested for response to each of the four components separately, they were found to respond almost exclusively to one component of the S+. These data are widely cited to support the view that discrimination learning in animals is a highly selective process. However, a subsequent experiment by Farthing \& Hearst (1970) importantly qualifies such an interpretation and helps to account for the discrepancy between such observations of dimension selectivity and the outcome of the present experiment. Farthing and Hearst trained pigeons in the manner of Reynolds' experiment, but tested the Ss on compounds of the training cues as well as on the components in isolation. They found selective dimensional control on the component tests, similar to that observed by Reynolds, but the tests with compounds showed that response was controlled by the reinforced compound as well. Thus, dimensional vs compound control of discriminative response is not an all-or-none matter for a given organism, and it seems reasonable to assume that the predominant form of stimulus control depends upon the nature of the test conditions imposed.

\section{REFERENCES}

EDW ARDS, A L. Experimental design in psychological research. (3rd ed.) New York: Holt, Rinehart, \& Winston, 1968.

FARTHING, G. W., \& HEARST, E. Attention in the pigeon: Testing with compounds or elements. Learning \& Motivation, 1970, 1, 65-78.

FELLOWS, B. J. Chance stimulus sequences for discrimination tasks. Psychological Bulletin, 1967, 67, 87-92.

GRAF, V., \& TIGHE, T. Subproblem analysis of discrimination shift learning in the turtle (Chrysemys picta picto). Psychonomic Science, 1971, 25, 257-259.

KENDLER, H. H. Environmental and cognitive control of behavior. American Psychologist, 1971, 26, 962-973.

REYNOLDS, G. S. Attention in the pigeon. Journal of the Experimental Analysis of Behavior, 1961, 4, 203-208.

SUTHERLAND, N. S. Stimulus analysing mechanisms. In Proceedings of $a$ symposium on the mechanisation of thought processes. Vol. 2. London: Her Majesty's Stationery Office, 1959.

TIGHE, T. J., GLICK, J., \& COLE, M. Subproblem analysis of discrimination shift learning Psychonomic Science, $1971,24,159-160$.

TIGHE, T. J., \& FREY, K. subproblem analysis of discrimination shift learning in the rat. Psychonomic Science, 1972, 28, 129-133.

ZEAMAN, D., \& HOUSE, B. J. The role of attention in retardate discrimination learning. In N. R. Ellis (Ed.), Handbook of mental deficiency. New York: McGraw-Hill, 1963. Pp. 159-223. 